\title{
A Review of Non-Destructive Damage Detection Methods for Steel Wire Ropes
}

\author{
Ping Zhou ${ }^{1}$, Gongbo Zhou ${ }^{1}{ }^{1} \mathbb{D}$, Zhencai Zhu ${ }^{1}$, Zhenzhi He ${ }^{2}$, Xin Ding ${ }^{1}$ and Chaoquan Tang ${ }^{1}$ \\ 1 Jiangsu Key Laboratory of Mine Mechanical and Electrical Equipment, School of Mechatronic Engineering, \\ China University of Mining and Technology, Xuzhou 221116, China \\ 2 School of Mechanical and Electrical Engineering, Jiangsu Normal University, Xuzhou 221116, China \\ * Correspondence: gbzhou@cumt.edu.cn; Tel.: +86-182-0520-7100
}

Received: 22 May 2019; Accepted: 5 July 2019; Published: 9 July 2019

\begin{abstract}
As an important load-bearing component, steel wire ropes (WRs) are widely used in complex systems such as mine hoists, cranes, ropeways, elevators, oil rigs, and cable-stayed bridges. Non-destructive damage detection for WRs is an important way to assess damage states to guarantee WR's reliability and safety. With intelligent sensors, signal processing, and pattern recognition technology developing rapidly, this field has made great progress. However, there is a lack of a systematic review on technologies or methods introduced and employed, as well as research summaries and prospects in recent years. In order to bridge this gap, and to promote the development of non-destructive detection technology for WRs, we present an overview of non-destructive damage detection research of WRs and discuss the core issues on this topic in this paper. First, the WRs' damage type is introduced, and its causes are explained. Then, we summarize several main non-destructive detection methods for WRs, including electromagnetic detection method, optical detection method, ultrasonic guided wave detection method, and acoustic emission detection method. Finally, a prospect is put forward. Based on the review of papers, we provide insight about the future of the non-destructive damage detection methods for steel WRs to a certain extent.
\end{abstract}

Keywords: non-destructive damage detection; steel wire ropes; review; electromagnetic detection; optical detection; ultrasonic guided wave

\section{Introduction}

Steel wire rope was invented in 1834. It has many advantages, such as high tensile strength, stable and reliable operation, and a strong capacity for dynamic load and overload. It is widely used in lifting, transportation, and traction equipment/systems. The main equipment/systems include mine hoists, ropeways, cranes, oil rigs, elevators, and cable-stayed bridges [1-4]. During the service of steel wire ropes (WRs), defects and damages like wire breakage, wear, rust, fatigue, strand breakage, and even sudden breaking will inevitably occur due to various reasons. Therefore, academia and industry have been trying to explore various methods to detect damages of WRs to guarantee its reliability and safety [5].

However, due to the complexity of WR structure, the diversity of working environment, and the limitation of detection methods, non-destructive detection of WRs has become an old and difficult problem [5]. The development of related technologies is slow, and it is difficult to achieve efficient and reliable industrial application. At present, manual inspection under low-speed operation (visual inspection combined with manual touch) is still the main method in most cases, and the electromagnetic detectors are used in a few cases, which cause problems such as missed detection, false detection, and failure to detect in time, resulting in frequent occurrence of faults and accidents [6]. Taking the mining industry as an example, on 29 December 2010, a wire rope at a resort in Maine broke and more 
than 200 people in the cable car were trapped in the air and 9 people were injured; On 9 March 2017, a wire rope of a multi-rope friction mine hoist of the Mining Company of Heilongjiang Longmei Group suddenly broke during the lifting process causing a cage fall (cable fire aggravates wire rope breakage). As a result, 17 people trapped in the cage were killed. Therefore, it is of great significance to study reliable and efficient non-destructive detection methods for WRs.

There are many methods for WRs damage detection [7], including electromagnetic detection, optical detection, ultrasonic guided wave detection, acoustic emission detection, ray detection, eddy current detection, and vibration detection. The most important methods are electromagnetic detection and optical detection (machine vision method). In particular, the instruments based on electromagnetic detection have been gradually applied in the market. Although the optical detection method can intuitively grasp the surface morphology characteristics of WRs, it is seldom used because of the influence of algorithm performance and the inability to detect internal defects. In addition, other methods are still in the theoretical and laboratory stage for many reasons. Non-destructive detection of WRs is an important research field, and there are some literature reports on various methods. However, there is still a lack of a reasonable and systematic summary to illustrate the current research situation and point out the possible research directions in the future.

Based on this, we first summarize the research status of each important detection method and analyze its shortcomings, then we summarize the existing methods and put forward the prospects. To our best knowledge, this article will make up for the lack of literature review in the field of non-destructive detection of WRs, which can bridge this gap and promote the development of non-destructive detection technology of WRs. The contributions of this paper can be summarized as follows: (1) The literatures in the field of non-destructive testing of wire rope are summarized in detail, including electromagnetic detection method, optical detection method, ultrasonic guided wave method, and acoustic emission method. (2) Shortcomings of these methods are pointed out, and comparisons are made among them. (3) Each important detection method is prospected in combination with the current technological situation, which is expected to promote the development of this direction.

The work is arranged as follows: In Section 2 the WRs damage type is introduced and its causes are explained. In Section 3 several main WRs non-destructive detection methods are reviewed. Their main disadvantages are summarized in Section 4. The prospect is presented in Section 5.

\section{Damage Types of Wire Ropes}

Steel wire ropes will be damaged in various forms when used, which will reduce the strength of the WRs and pose a potential threat to the system safety. Various damage conditions and distribution have different effects on the strength reduction of WRs, and the whole WR is often scrapped due to a certain section of the WR. Therefore, the study of various damages of WRs will be beneficial to the correct evaluation of its state [8,9]. The damage types of WRs include wire breakage, wear, deformation, rust, and fatigue. Among them, fatigue has a variety of representations, such as internal and external cracks, internal and external wire breakages, and slack. The common types are shown in Figure 1. 


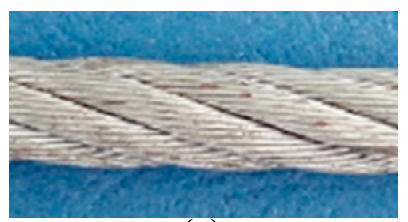

(a)

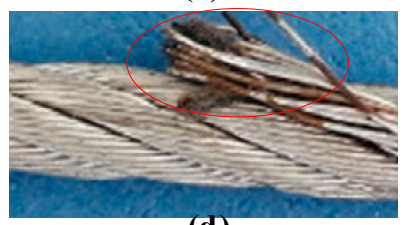

(d)

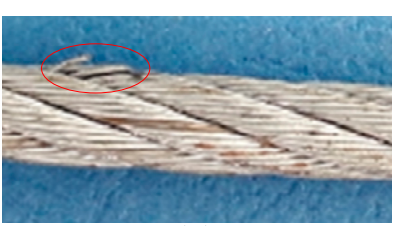

(b)

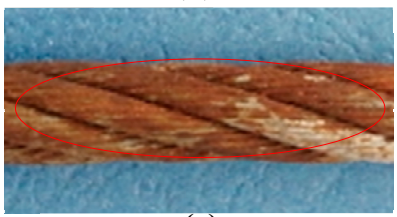

(e)

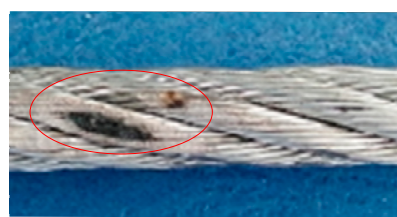

(c)

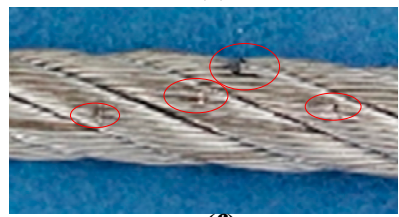

(f)

Figure 1. Sample pictures of wire rope damage. (a) Health; (b) wire breakage; (c) wear; (d) deformation (broken strand); (e) rust; and (f) fatigue (fatigue breaking).

In practice, the above damage types will interact with each other, such as rust will aggravate the wear process, wear will promote wire breakage, fatigue will promote the generation or aggravate the evolution of wire breakage and wear, and the comprehensive impact of wear, wire breakage, fatigue, and rust will promote broken strands and aggravate the scrap of wire rope. When minor faults evolve to a certain extent or suffer sudden impact load, strand breakage will occur, which will obviously affect the life of WR. Because of the different working conditions and environment, the development speed and degree of damages are also different. In current standards, the residual strength is generally obtained by finding the defects of WR in service to infer the strength loss, then, whether the WR is scrapped is judged according to the safety factor of WR and the rules of use [9].

The description of different damage types of WRs is shown in Table 1.

Table 1. Types and description of wire rope (WR) damage.

\begin{tabular}{cl}
\hline \multicolumn{1}{c}{ Types } & \multicolumn{1}{c}{ Description } \\
\hline Wire breakage & $\begin{array}{l}\text { Fatigue breakage and wear breakage are the main causes of wire } \\
\text { breakage. Wire breakage will reduce the WR strength and increase the } \\
\text { potential safety hazards of WRs. }\end{array}$ \\
\hline Wear & $\begin{array}{l}\text { Wear is one of the most common phenomena of WR, which usually } \\
\text { evolves from normal wear to failure wear. The wire wear will reduce the } \\
\text { tension it can bear. }\end{array}$ \\
\hline Rust & $\begin{array}{l}\text { Rust is a fault phenomenon of WRs caused by the chemical and } \\
\text { electrochemical action of surrounding medium, which has a significant } \\
\text { impact on the life of WRs. }\end{array}$ \\
\hline Fatigue & $\begin{array}{l}\text { Impact on the WR causes damages (including flattening, strand } \\
\text { relaxation, kinking, bending, and strand breakage) to the original } \\
\text { structure, which accelerates the scrap. }\end{array}$ \\
\hline & $\begin{array}{l}\text { After repeated bending for a certain number of times or repeated } \\
\text { stretching and twisting, fatigue occurs, which leads to changes in the } \\
\text { internal and external performance of the WR. }\end{array}$ \\
\hline
\end{tabular}

In general, in the WR damage detection and diagnosis practice, according to the characteristics of WR damages, the WR damages can be divided into two categories [10]: local flaw damage type (LF) and loss of metallic cross-sectional area type (LMA). LF type refers to the damage locally generated in the WR, mainly including internal and external breakage, corrosion pit, and deep wear of steel wire, characterized by a sudden decrease in the metallic cross-sectional area of the WR; LMA type refers to the slow reduction of the effective metallic cross-sectional area in the long range along the axial direction of the WR, which mainly includes wear, long-distance rust, and rope diameter reduction. 


\section{Non-Destructive Detection Method for Steel Wire Ropes}

The following is a literature review of electromagnetic detection, optical detection, ultrasonic guided wave, acoustic emission detection method, and other detection methods.

\subsection{Electromagnetic Detection Method}

Electromagnetic detection is one of the oldest methods in non-destructive testing field and has been applied earliest to WRs detection. In this detection method, there are two different excitation modes including coil excitation and permanent magnet excitation, and the commonly used sensor types are the Hall sensor and the induction coil.

The detection principle diagram is shown in Figure 2, the main principle is: after the WR is excited by coil or permanent magnet, the surface and internal damages will change the magnetic flux in the WR. Then the signal is detected by the induction coil or integrated sensor (e.g., Hall sensor and magnetoresistance sensor), and the type or degree of damages can be obtained to a certain extent through further processing and analysis of the signal [8-10]. Figure $2 \mathrm{a}$ is the main magnetic flux detection method based on coil excitation, which adopts the coaxial magnetization method, namely, taking the measured wire rope as the core, and the wire rope segment between the two coils is magnetized by the coil with current. Figure $2 b$ is the main magnetic flux detection method based on permanent magnet excitation, in which the method of inter-pole magnetization is adopted, taking the wire rope as a component of the magnetic circuit, and the induction coil is used to detect the change of magnetic flux inside the wire rope caused by damage. Figure $2 \mathrm{c}$ is a method of return magnetic flux detection based on permanent magnet excitation, whose excitation mode is the same as Figure $2 b$, but the Hall sensor is used to detect the flux density in the magnetic circuit formed by excitation device, air gap and wire rope. Figure $2 \mathrm{~d}$ is leakage magnetic flux detection method based on permanent magnet excitation, whose excitation mode is the same as Figure $2 b, c$, however, the difference is that the sensor is used to collect the leakage magnetic field information caused by the damage.

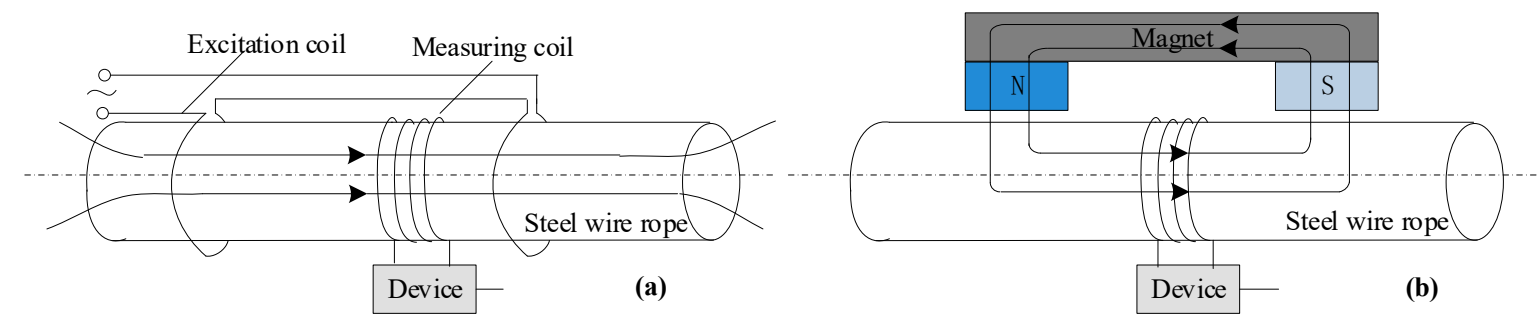

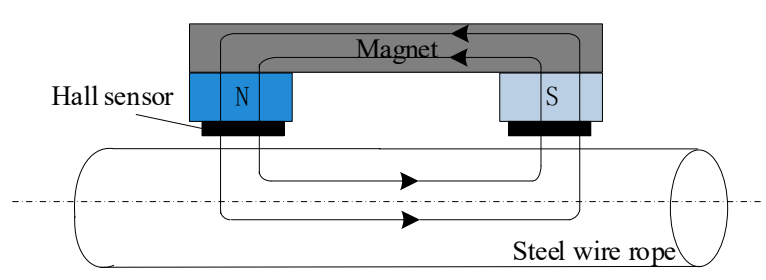

(c)

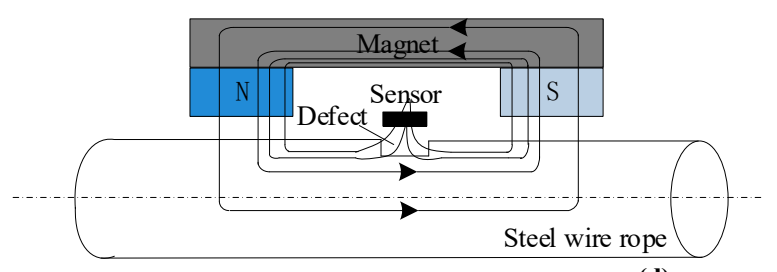

(d)

Figure 2. Electromagnetic non-destructive detection principle of steel wire rope. (a) Main magnetic flux detection method based on coil excitation; (b) main magnetic flux detection method based on permanent magnet excitation; (c) return magnetic flux detection method based on permanent magnet excitation; and (d) leakage magnetic flux detection method based on permanent magnet excitation.

In 1906, South Africa developed the first non-destructive testing device for WRs based on electromagnetic principle. Over the past 100 years, electromagnetic detection technology has made great progress [11]. The research of electromagnetic detection technology mainly includes: the mechanism of electromagnetic detection signal under the influence of defect parameters (defect width, depth, number of broken wires, etc.) or the theoretical model of the relationship among them; the magnetization 
method and device of WRs; the electromagnetic signal detection means such as the coil sensor, Hall sensor, and magnetoresistance sensor. It is noted that the signal processing and recognition methods are accompanied with the above three aspects. Following is a literature review of the above three aspects.

\subsubsection{Mechanism and Model}

For electromagnetic detection mechanism and theoretical model, many studies have been carried out by researchers at home and abroad.

For instance, Norouzi et al. [12] used the finite element analysis method to optimize the pole shoe length, and the optimized results help make the magnetic flux distribution in the measured parts as uniform as possible, which was more conducive to magnetic leakage detection. In [13], Zhao et al. performed finite element analysis for the magnetic flux leakage (MFL) distribution of WR typical defects based on strong magnetic detection method and obtained the relationship between MLF signal and detection distance, damage depth, damage axle width and internal wire breakage. In [14], Lenard et al. paid attention to oil and gas pipelines, and analyzed the influence of line pressure, bending and residual stresses on MFL signal in MFL detection process through experiment and finite element simulation. To study the effects of probes and probe lift-off on the defect leakage magnetic field, Krzywosz [15] and Kalwa et al. [16] respectively carried out research and proposed that the smaller the lift-off value of magnetic sensitive probes, the better.

In order to apply the magnetic dipole to the three-dimensional magnetic field modeling of MFL, Dutta et al. [17] first presented a three-dimensional (3D) magnetic leakage model of ferromagnetic materials surface fracture based on magnetic charge theory. Based on [17], an improved 3D MFL model for numerical analysis is proposed by Trevino et al. [18], which can be used to detect surface fracture of ferromagnetic materials under saturated magnetization. To further explain the formation principle of MFL, Sun et al. [19] explained the mechanism of MFL in engineering from the perspective of magnetic refraction, and found that the signal component in defect detection was different from that in traditional MFL analysis, which was produced by protruding defects. Considering the effect of tensile stress on the strength of the MLF of a steel WR defect, Gao et al. [20] loaded a variable force onto reproduced samples of typical defective WRs relying on an inspection platform. The experimental results showed that the peak-to-peak values of the MFL signal from all defective WRs increased with tensile stress, in an approximately linear relationship.

\subsubsection{Magnetization Method}

The magnetizer is an important component of WR non-destructive detection system. Its excitation structure and mode will produce different magnetization effects [21]. The commonly-used magnetization methods mainly include the coil electromagnetic magnetization method and permanent magnetization method.

Thus far, Kang et al. [22] analyzed the different WR magnetization effect of different magnetization methods by finite element method and obtained the optimum size of feed-through magnetizer. In [23], the equipment was operated according to the principle of strong magnetic detection, which realized magnetization saturation by magnetizing the wires with a pre-magnetic head. Jomdecha et al. [24] improved the traditional current excitation device, so that the excitation intensity of the device can be controlled by adjusting the excitation power supply and the coil. Wang et al. [25] designed an excitation device that can restrain the fluctuation of lift-off distance and can improve the structure of the detection device. Song et al. [26] studied whether AC magnetization in U-yoke would cause eddy current disturbance field. The results showed that the axial component of MFL of crack was independent of excitation frequency and intensity.

In the practical application, for thin WR in strong electromagnetic interference environment, Yan et al. [27] proposed an electromagnetic non-destructive testing method. In this method, a simplified magnetic circuit was proposed to excite WR, and the defect detection of thin WR in electromagnetic 
interference environment was realized. A new WR tester was established based on MLF principle in [28], and each arc segment subtends an angle of 22.5 degrees at the center by cutting two rings of $\mathrm{NdFeB}$ along axial direction into 32 equal arc segments, which were then parallelly magnetized in magnetizer. To detect WR damage in coal mine, Sun et al. [29] proposed an opening electric magnetizer through the magnetic control for a C-like electric loop-coil, which has an opening structure capable of directly encompassing and centering the endless object in it. The magnetizer can solve problems, i.e., installation inconvenience and on-line detection difficulty in the non-destructive testing of coal mine wire rope fixed at both ends.

\subsubsection{Detection Sensor}

(1) Coil sensor

Coil sensor is widely used because of its low cost and ease of use. In recent years, its application in WRs non-destructive detection is still further studied.

For example, Liu et al. [30] assessed the surface and internal flaws using a new type of sensor to measure the biased pulse magnetic response in a large-diameter steel stay cable. Two parallel connected flexible flat coils fed with a biased pulse current were deployed by the sensor as the electromagnet for cable magnetization. Yan et al. [31] put forward a kind of iron core as coil winding skeleton for the wire rope non-destructive testing relying on the theoretical analysis and 3D transient magnetic field simulation. The experiment results proved that the signal to noise ratio of coil with the iron core proposed in this paper increased almost six times, making it easier for defect analysis. Aiming at detecting the change of surface leakage magnetic field, Fedorko et al. [32] designed a pair of induction coils of different sizes and analyzed the static magnetic field distribution based on finite element simulation. Sun et al. [33] designed a new MLF sensor based on open magnetizing method. Experimental comparisons between the open and yoke probes for on-line automated monitoring were conducted, which confirmed the characters of smaller magnetic interaction force, less wear, and damage in this method were in contrast to the traditional on-line automated structural health monitoring technology.

(2) Integrated sensor

Through using integrated sensors to detect MLF of the WR surface, the circumferential and axial locations of defects can be founded, so as to analyze whether the defects belong to centralized or decentralized wire breakage, and to make a further accurate judgment on the damage of WRs [11].

In the work of [34-38], the Hall sensors were used. Xu et al. [34] designed a sensor module based on Hall sensor to test the leakage magnetic field of stay cables online and compared three different filtering algorithms to obtain the leakage magnetic field of artificial damage inside the cable. Wang et al. [35] proposed a new method to detect magnetic excitation in WRs by combining the structural models for dynamic magnetic field balancing and magnetic focusing. A Hall-element array and the magnetic focusing technique were used to reduce the interference produced by the interactions between the environmental magnetic fields and the wire rope strand. A MLF method is adopted by Kim et al. [36] for the detection of local damage when inspecting WRs, and a multi-channel MFL sensor head was fabricated by adapting to the wire rope with a Hall sensor array and magnetic yokes, the accuracy and reliability were evaluated based on the comparison of the repeatedly estimated damage size and the actual damage size. Tian et al. [37] designed an optimal model for magnetic excitation to develop a non-destructive sensor using a Hall-effect sensor for coal mine hoist wire ropes. Then, a new detection method called the permanent magnet co-directional excitation flux-weakening method was put forward in [38]. The emulation and experimental results revealed that the defect detection sensor proposed in the paper can improve the magnetic signal from the defect by six to eight times.

In addition, the magnetoresistance sensors were used in the work of [39,40]. Singh et al. [39] proposed giant magnetoresistive-based MLF technique for condition monitoring of $64 \mathrm{~mm}$-diameter steel track rope which can be used to transport coal. Two saddle coils were used to magnetize the rope 
in this technique, and a giant magnetoresistive sensor was able to detect the tangential component of leakage magnetic flux from flaws. In addition, the technique can detect $2 \mathrm{~mm}$-deep flaws with good signal-to-noise ratio and solve the flaw whose interval is more than $3.2 \mathrm{~mm}$. Tunnel magnetoresistive (TMR) devices was first employed to form a circular MLF sensor by Liu [40], aiming to detect slight WR flaw. Two versions of this tailor-made circular TMR-based sensor array were put forward to inspect WRs with the diameters of $14 \mathrm{~mm}$ and $40 \mathrm{~mm}$, respectively.

\subsection{Optical Detection Method}

Optical detection method is an efficient non-destructive detection method, which has been developed in the field of WR surface damage detection. It can intuitively grasp the damage situation of WR surface. At the same time, with the development of WR oil pollution removal method/device [41], the influence of oil pollution gradually decreases.

The principle of optical detection method is shown in Figure 3, which generally includes two parts: image acquisition and damage diagnosis. The image acquisition generally uses high-speed camera to collect the surface image of WRs, and the damage diagnosis includes image preprocessing and pattern recognition. WR damage detection based on pattern recognition is a potential research direction. Specifically, the hardware and software of the image acquisition system are configured according to the actual situation; the collected images are first processed by pretreatment method like cutting or filtering. Then, feature extraction methods (such as local binary pattern and gray level co-occurrence matrix) are used to extract features, and next dimensionality reduction is carried out on the established feature datasets. Finally, machine learning classifiers (such as support vector machines, artificial neural networks, etc.) are used for training and testing to obtain robust classifiers for state recognition of unknown images [42].

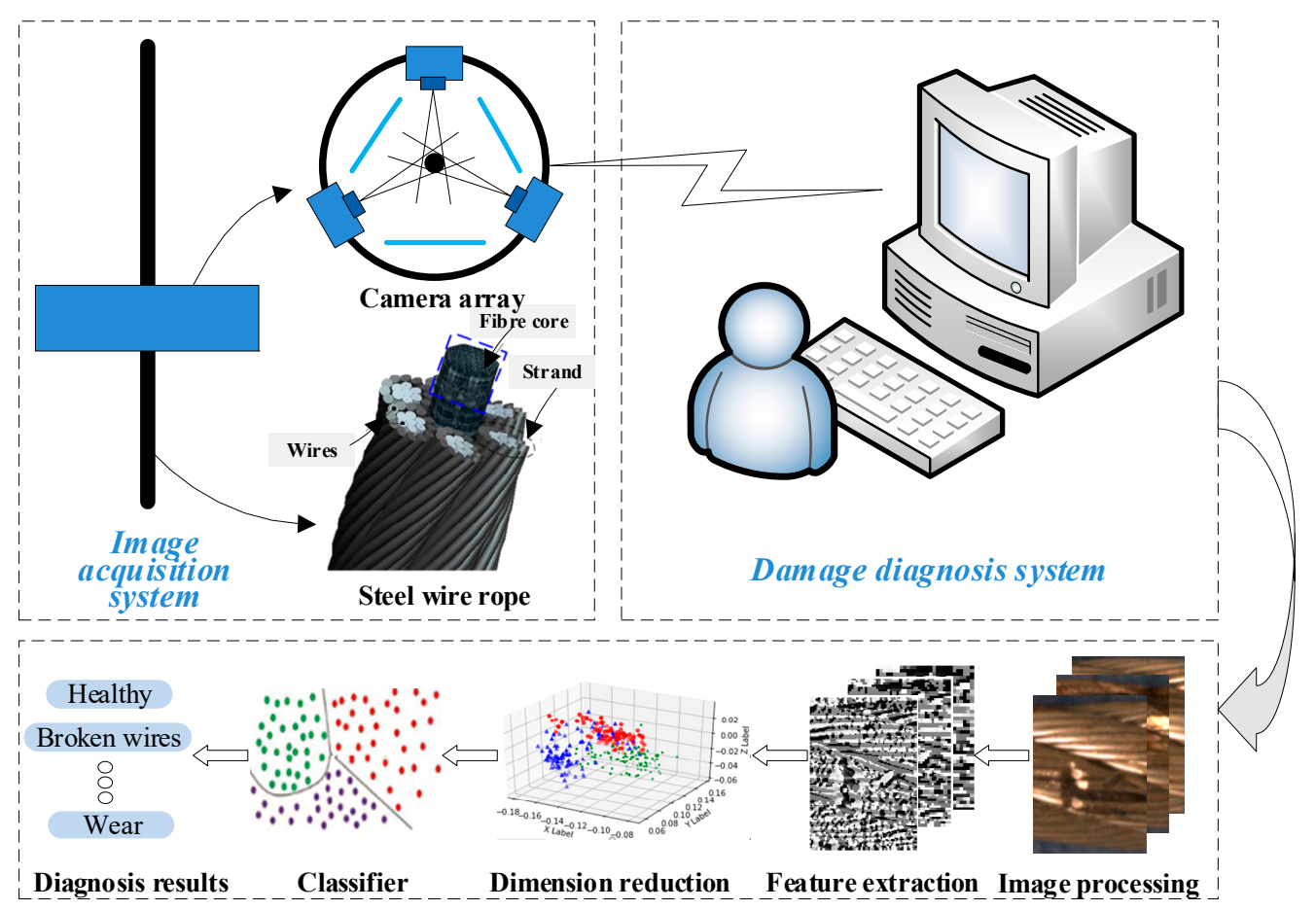

Figure 3. Optical detection principle for steel wire rope.

Based on camera and offline processing algorithm, Vallan et al. [43] established a measurement system which measured the change of rope length according to the characteristics of rope twist to detect the damage of rope. For elevator rope, Yaman et al. [44] proposed a fault monitoring method based on image processing and autocorrelation analysis, which realized the defect detection of steel 
rope to a certain extent. In [45], Sun et al. realized the identification and segmentation of WRs boundaries through image processing and clustering methods. Platzer et al. [46] proposed using Hidden Markov field model to locate the defects of WR, the results demonstrated that the detection performance of this method is better than that of the previous time invariant system classification method. Then, they compared the performance of different texture features in the detection of WR surface defects in [47]. For the defects of WR, the histogram of oriented gradient feature was the best, followed by the local binary patterns feature. In the work of $[48,49]$, Wacker et al. established a probabilistic appearance model as a representation of normal surface changes combined with the structure and appearance of the WR and realized the detection of WR abnormal surface. For surface defects of thin metallic wires, an automatic optical detection technique was presented by Sanchez-Brea et al. in [50]. This technique was based on the intensity variations on the scattered cone generated when the wire is illuminated with a beam at oblique incidence.

\subsection{Ultrasound Guided Wave Method}

Ultrasonic guided waves method (UGW) is one of the promising methods for investigation of non-homogeneities for the internal structure of WRs [51]. The measurement schematic diagram is illustrated in Figure 4, which is consisted of the computer, the ultrasonic measurement system, the preamplifier, the transmitter, and the receiver, detailed principles are available for reference [52]. The wave is launched on WRs and propagates along the strand of WRs. Acoustic reflection occurs when the sound wave passes through the damage. After collecting and analyzing the echo at the receiving end, the damage inside and outside the WR can be obtained [53].
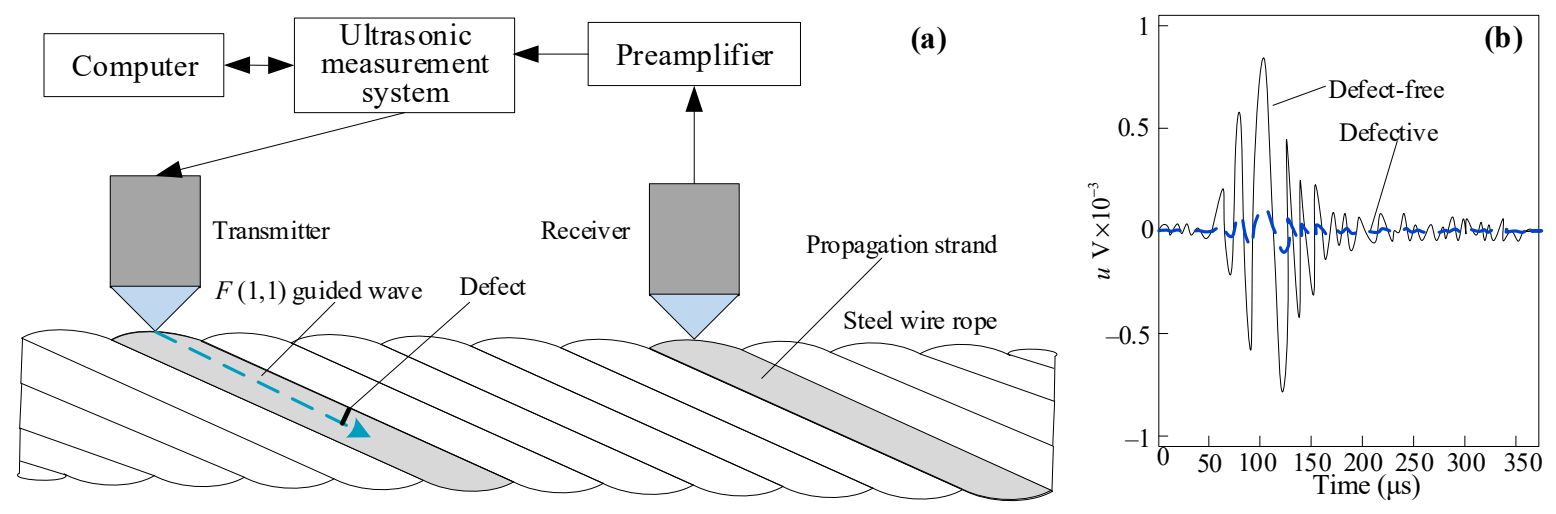

Figure 4. Non-destructive testing principle of steel wire rope by ultrasonic guided wave method [52]. (a) Detection system schematic diagram; (b) sketch map for waveforms of defect-free and defective signal.

For purpose of the generation and reception of ultrasonic longitudinal guided waves in seven-wire steel strands in a pitch catch arrangement, Liu et al. [53] optimized the magnetostrictive transducers configuration for both transmitter and receiver. Consequently, magnetostrictive transducers with an optimized configuration, including permanent magnet distribution and multilayer coil connection, could be efficiently used for the inspection of seven-wire steel strands by using UGW in a pitch catch arrangement. In [54], Treyssede et al. studied the transmission characteristics of elastic guided wave in multi-stranded WR. The dispersion curve of spiral WR was obtained by semi-analytical finite element method and signal energy calculation, and the optimum excitation and receiving position of ultrasonic guided wave was calculated. Vanniamparambil et al. combined UGW, acoustic emission technology and digital image correlation, and fused the features obtained by the three technologies to achieve non-destructive testing [55]. Xu et al. [56] studied the effect of different frequencies of UGW on the detection rate of steel WR defects. It was found that the higher frequency of guided waves in the steel WR lead to the faster energy attenuation, and the receiving length of elastic waves increased with the frequency. The propagation characteristics of various UGW between strands and 
cores of WR and their effects on the penetration depth of WR were studied in [57] by Raisutis et al. In the work of [58], the efficiency of employing the magnetostriction of ferromagnetic materials were studied relying on the UGW method for WRs damage inspection, and the location and severity of damages were approximately identified and characterized using the short-time Fourier transform and wavelet analysis.

\subsection{Acoustic Emission Detection Method}

Acoustic emission (AE) refers to the transient elastic wave phenomena produced by the rapid release of energy in the local area of the material under the influence of the outside world [59]. Acoustic emission sensors can convert transient elastic waves into electrical signals based on piezoelectric effect. The change of internal damage can be inferred from the analysis of electrical signals [60]. The AE testing system is shown in Figure 5a (in practical application, specific device structure and detection method should be designed according to specific detection objects) [61]. The testing principle are as follows: the two ends of the WR specimen are installed on the tensile test bench, and two clamps are clamped in the middle of the WR to help install two acoustic emission sensors. The loading parameters of the test bench are controlled by a load controller. Acoustic emission equipment is used to collect and analyze the collected data in the process of loading to obtain the occurrence and location of defects on the WR. The waveform of broken wire is shown in Figure $5 \mathrm{~b}$. $\mathrm{AE}$ technology can qualitatively identify the broken wire signal, which has the characteristics of high amplitude and high absolute energy [62]. However, because this method can only be used in the static load part, the signal-to-noise ratio is low, the cost of the instrument is high, and it is difficult to measure dynamically, it is still in the laboratory stage at present, and is difficult to be effectively used in engineering.
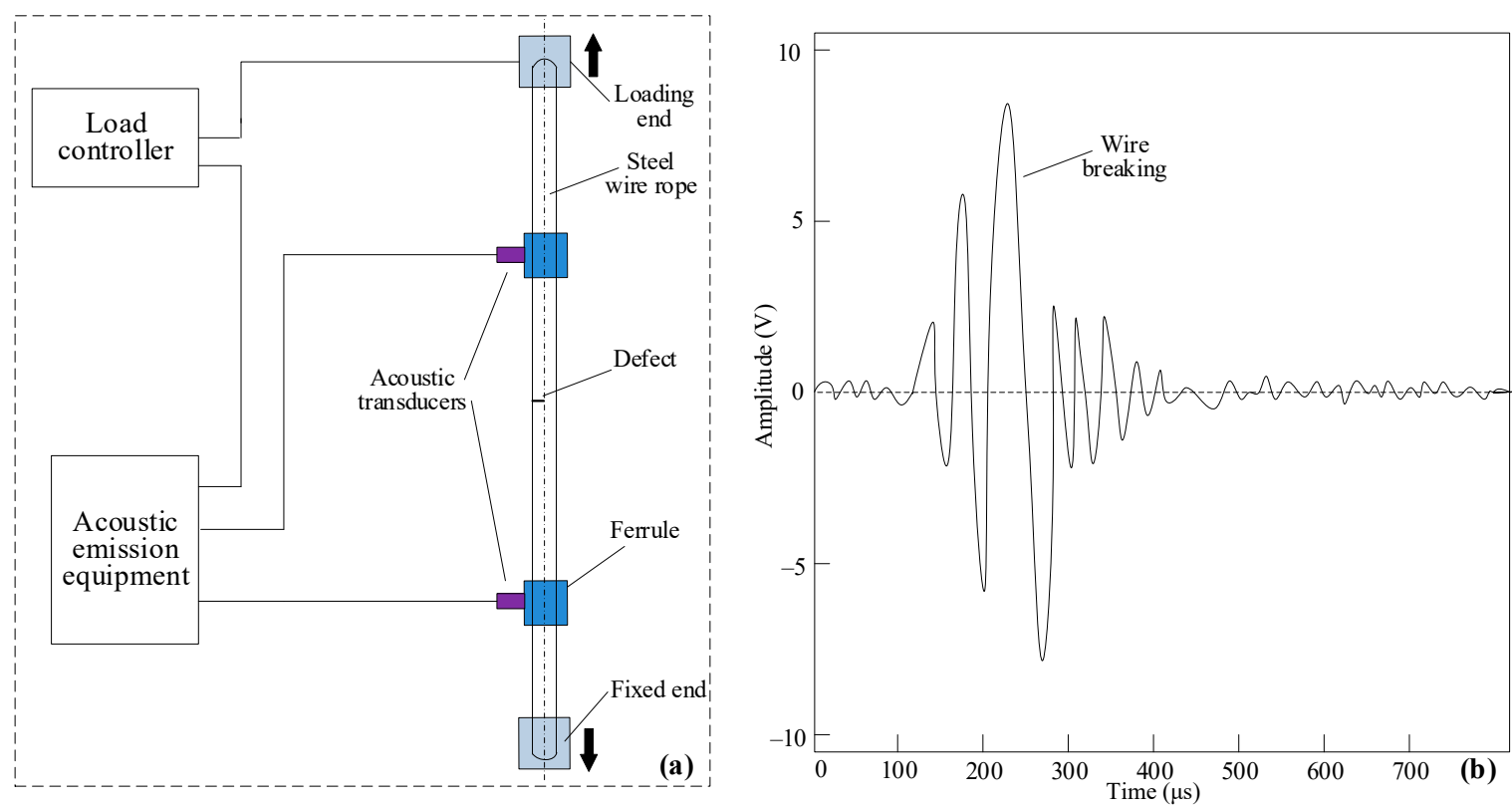

Figure 5. Acoustic emission detection for steel wire rope [61]. (a) Detection system schematic diagram; (b) sketch map for waveform of defects and wire breaking signal.

The research of relevant experimental methods and principles is active. The most systematic research on detecting broken wire of WR by AE technology was carried out by Casey et al. [63-67] in the 1980s. Based on a large number of experiments, they put forward an analysis method of amplitude distribution, that is, using the amplitude of AE signal to judge wire breakage. Due to the limitation of instrument level at that time, the result was not very ideal. Casey et al. [68] summarized the research of $\mathrm{AE}$ technology in the defect monitoring of WR at first, pointed out that the most important 
application of AE technology in the WR monitoring is the detection and location of broken wires, and discussed in detail the influence of the WR structure, the size and the number of broken wires on the AE monitoring results. Subsequently, with the development of technology, this method has been further studied. AE technology was adopted to quantitatively detect wire breakage in WR by Shao et al. [69]. The appropriate timing parameters were determined by waveform analysis, and the broken wire event was characterized as a single AE impact signal, which realized the quantitative expression of broken wire. For the problem of that inter wire fretting in ropes could cause excessive low amplitude noise, a new method based on modern signal processing techniques proposed by Ding et al. [70] could be applied to solve it. Drummond et al. [61] monitored the fatigue process of wire ropes by $\mathrm{AE}$ and established the relationship between the $\mathrm{AE}$ signal characteristics and wire breaks. Bai et al. [62] used AE technique to monitor the tensile testing process for two kinds of elevator wire ropes, and in this work AE signals from wire breaks were obtained and analyzed by AE parameters and waveforms. In the work of [71], Li et al. proposed an innovative monitoring method that used waveguides to draw out the steel wires at the end of the cable, which can effectively capture the AE signal produced by defects/wire breaking and accurately locate the transverse position of the wire breaking of the cable. Li et al. [72] developed a damage assessment and warning method for stay cables based on the AE technique and fractal theory. In this work, the fatigue test of composite cable was conducted, the fractal dimension of AE signal was analyzed, the damage index according to the fractal dimension was established.

\subsection{Other Detection Methods}

Only a few literature reports involve other non-destructive detection methods of WRs, such as eddy current detection [73,74] and ray detection [75,76], and are in the laboratory stage for many reasons. The detection principles are depicted in Figure 6. Figure 6a represents the eddy current method and Figure $6 \mathrm{~b}$ explains the ray method.
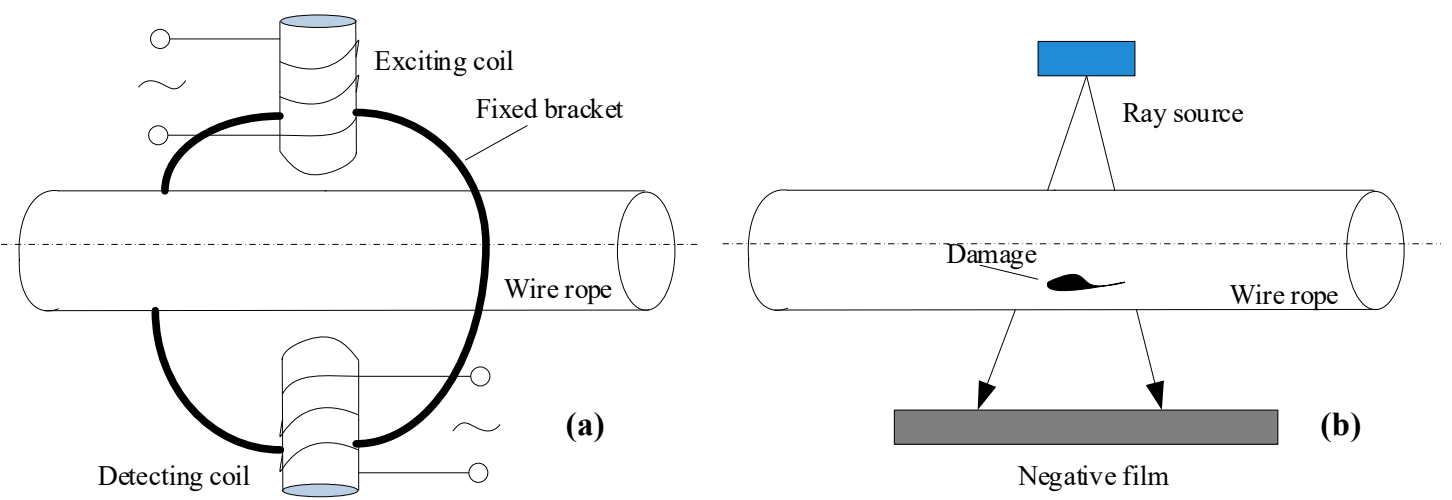

Figure 6. The other detection method principles. (a) Eddy current method [74]; (b) ray method [75].

Eddy current non-destructive testing technology takes eddy current effect produced by metal conductor in alternating magnetic field as its working principle. It has the advantages of high sensitivity, fast detection speed, non-contact, etc. [73]. Using low frequency transmission eddy current testing method, Cao et al. [73] designed an adjustable annular eddy current testing device with symmetrical probe arrangement along the radial direction to perform the quantitative detection analysis of WR breakage defects. Meanwhile, Cao et al. [74] developed an experimental eddy current sensor and computer measuring system to obtain characteristic data for rope samples made in laboratory. The characteristic data are identified by the RBF network, and the identification results show the proposed evaluation method is feasible.

When non-destructive testing of WR is carried out by ray method, the damage situation inside and outside of the WR can be clearly presented on the negative film because of the different absorption ability of radiation rays between the surface/internal defect and non-defect of the WR $[75,76]$. For this reason, 
Zhang et al. [75] proposed a new WR detection system based on X-ray digital imaging technology, and introduced the X-ray digital imaging technology, system structure and working principle. A detection system of bridge cables using gamma ray is proposed by Peng et al. [76]. The exposure time and sensitivity of steel cables in gamma ray detection were studied. Meanwhile, the comparison between the ray detection method and the electromagnetic detection method was performed. The actual measurement demonstrated that the research results can improve the safety of WR and ensure the bridge safety assessment.

\section{Existing Shortcomings}

In summary, we find that each non-destructive detection method of WRs has its own advantages, but there are still many shortcomings $[9,77,78]$, as shown in Table 2.

Table 2. Comparison of several wire rope detection methods.

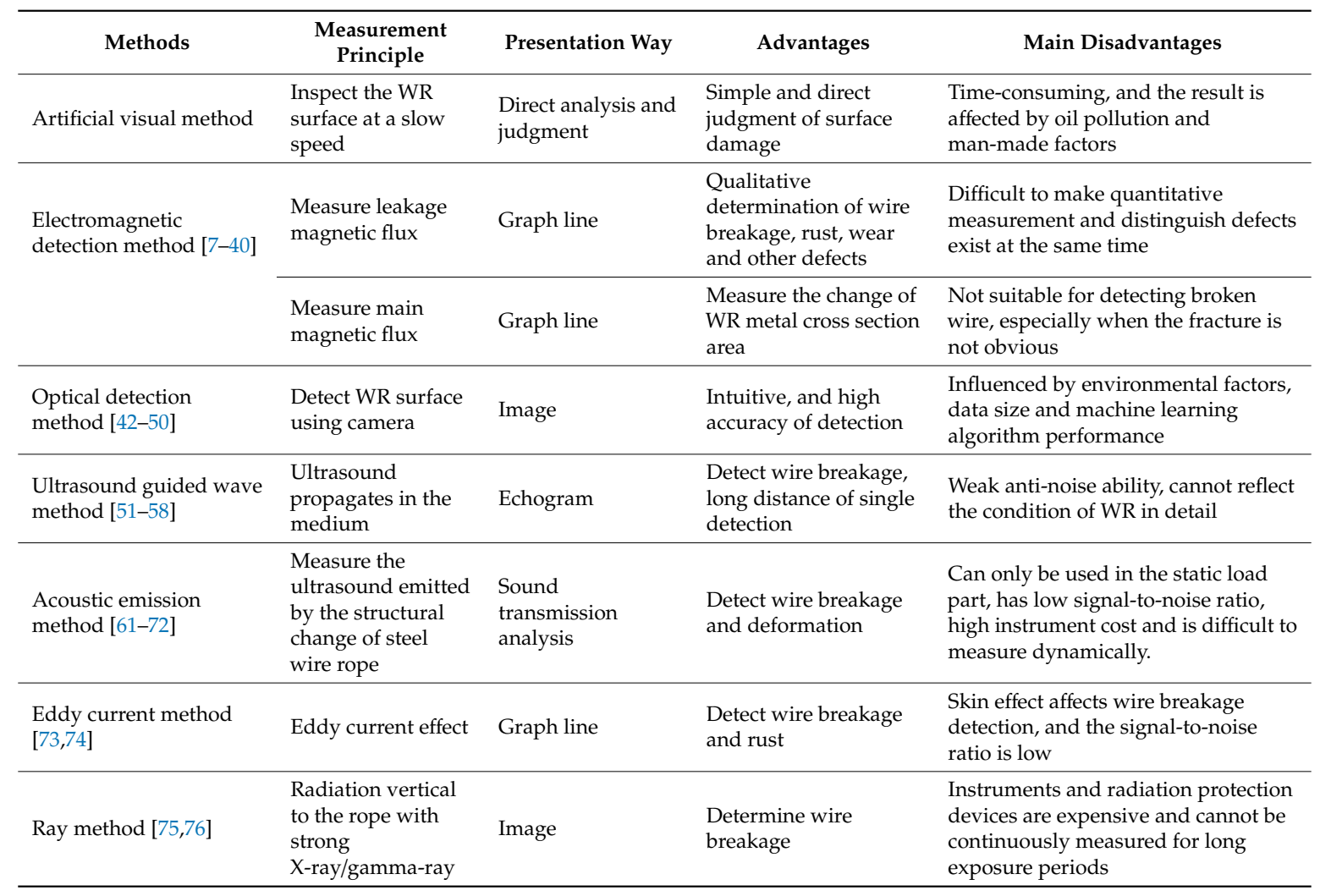

In view of the problems existing in electromagnetic detection, optical detection, ultrasonic guided wave method, acoustic emission method, and other detection methods, the following are specified in detail:

(1) In the field of WR electromagnetic detection, the existing theory and technology have developed in depth. However, according to the current research situation, the problems in the electromagnetic non-destructive detection of WR mainly include the influence of different WR structures on the detection signal, the quantitative analysis of LF type defects, the formulation of testing standards, and the relationship between LMA, LF defects and the strength of WRs (details can be found in reference [78]). Only by solving the quantitative detection of LF type defects and the relationship between defects and the strength of WRs, can the service life of WRs be truly and accurately predicted by non-destructive testing.

(2) In the field of WR optical detection, the existing research has a small amount of data, namely no big data sets, and the algorithm is with shallow structure. It has some problems such as limited 
excavation ability, low computational efficiency and poor robustness. It is difficult to carry out high-speed detection, high efficiency and reliable identification, therefore can not be directly applied to real-time state health monitoring of WR. By introducing the deep learning [79-82] method into the WRs damage detection/monitoring, it is expected to achieve real-time, reliable and accurate damage identification and location, thus realizing the detection and monitoring of WR surface damage [83].

(3) The ultrasonic guided wave method, acoustic emission method and other detection methods are still in the stage of theoretical research and laboratory, and there is still a certain distance away from practical application, due to the limitation of technical level and the method itself. UGW and AE method are difficult to be used in actual dynamic detection, and the signal-to-noise ratio needs to be further improved; Eddy current method is less studied and affected by skin effect. Its reliability needs further study; Ray Method's development prospects are relatively limited compared with other methods because of the expensive instruments and the potential impact of radiation on the human body. Only by further deepening theoretical and experimental research and solving more of the above difficult problems can we promote the progress of these detection methods.

\section{Summaries and Prospects}

To promote the development of non-destructive detection method for WRs, we present an overview of non-destructive damage detection methods for WRs in this paper. Some summaries are listed as follows:

(1) The types of WR damages and their causes were introduced in detail, including wire breakage, wear, rust, deformation, and fatigue. They are divided into two types: local flaw and loss of metallic cross-sectional area.

(2) The development status of several important detection methods including electromagnetic detection, optical detection, ultrasonic guided wave method, acoustic emission detection, eddy current detection, and ray detection was reviewed, and their advantages and disadvantages were compared and summarized. On the whole, electromagnetic detection method has gradually been applied in practice. Optical method has shown great potential for application, while other methods are still in the laboratory stage.

In addition, some research trends and potential future research directions are given as follows:

(1) Electromagnetic detection method: the influence of WR structure on electromagnetic signal is expected to be studied; to solve the quantitative detection of LF type defect, the relationship between LF type defect and electromagnetic signal characteristics should be further investigated; the relationship between defect and WR strength should be further determined through a large number of experimental studies, so as to achieve real and accurate prediction of WR service life; and relevant standards should be further improved and developed combining the research results with the practical experience.

(2) Optical detection method: methods to reduce or eliminate the influence of oil and light on the surface of WRs should be further designed; deep learning method is expected to be introduced because of its strong data mining ability, and more efficient, accurate, and robust algorithm than the traditional machine learning should be designed; to make the designed algorithm more robust, a big data set of WR surface defects with different WR types and different sizes (diameter, wire number) is expected to be established to cover all sample space.

(3) Other detection methods: theoretical and experimental research, especially ultrasonic guided wave method, acoustic emission method, eddy current detection method and ray detection method, should be further deepened, and the intrinsic relationship between detection methods and the respective parameters of WR is expected to be explored; at the same time, relevant detection equipment should be designed and optimized to promote the development of related technologies. 
(4) Comprehensive application: a promising avenue for research is to combine various methods to characterize and detect damages from multiple dimensions. For example, the combination of electromagnetic method and optical method can not only grasp the size of effective cross-sectional area of WRs, the types of internal and external damages in real time, but also intuitively master its surface morphology characteristics, so as to provide more valuable parameters for the WR health evaluation.

Although there are many issues and challenges, as new technologies/methods and algorithms are introduced, it is believed that this field will have a more and more prospective future and one day it will be possible to truly realize efficient and accurate non-destructive detection of WRs.

We believe that this review has synthesized individual pieces of information on non-destructive damage detection of steel WRs and has provided a comprehensive reference for these readers who are interested in this research field.

Author Contributions: Conceptualization, P.Z. and G.Z.; Formal analysis, P.Z., G.Z., Z.Z., and Z.H.; Funding acquisition, G.Z. and P.Z.; Investigation, P.Z., Z.Z., X.D., C.T., and Z.H.; Supervision, G.Z., Z.Z., C.T., and Z.H.; Writing-original draft, P.Z.

Funding: This work was supported by the National Key Research and Development Program of China (grant number 2016YFC0600905), by the Postgraduate Research \& Practice Innovation Program of Jiangsu Province (grant number KYCX19_2140) and Postgraduate Research \& Practice Innovation Program of China University of Mining and Technology (grant number KYCX19_2140), by the Jiangsu Provincial Outstanding Youth Fund of China (grant number BK20180033), by the National Natural Science Foundation of China (grant number 51575513), and by the Project Funded of the Priority Academic Program Development of Jiangsu Higher Education Institutions (PAPD).

Acknowledgments: Thanks for the journal editors for their kind invitation to present this feature article. We wish to thank the authors of all references for their outstanding contributions in this field. At the same time, the authors would like to thank all reviewers and editors for their constructive comments.

Conflicts of Interest: The authors declare no conflict of interest.

\section{References}

1. Mouradi, H.; Barkany, A.E.; Biyaali, A.E. Steel wire ropes failure analysis: Experimental study. Eng. Fail. Anal. 2018, 91, 234-242. [CrossRef]

2. Henao, H.; Fatemi, S.M.J.R.; Capolino, G.A.; Sieg-Zieba, S. Wire rope fault detection in a hoisting winch system by motor torque and current signature analysis. IEEE Trans. Ind. Electron. 2011, 58, 1727-1736. [CrossRef]

3. Peng, Y.X.; Chang, X.D.; Sun, S.S.; Zhu, Z.C.; Gong, X.S.; Zou, S.Y.; Xu, W.X.; Mi, Z.T. The friction and wear properties of steel wire rope sliding against itself under impact load. Wear 2018, 400, 194-206. [CrossRef]

4. Krešák, J.; Peterka, P.; Kropuch, S.; Novákb, L. Measurement of tight in steel ropes by a mean of thermovision. Measurement 2014, 50, 93-98. [CrossRef]

5. Yuan, F.; Hu, B.L.; Zhou, Z.J. An analysis on the research status quo and prospects of defect detection methods of wire ropes. Mach. Des. Manuf. 2010, 2, 260-262. [CrossRef]

6. Čereška, A.; Zavadskas, E.K.; Bucinskas, V.; Podvezko, V.; Sutinys, E. Analysis of steel wire rope diagnostic data applying multi-criteria methods. Appl. Sci. 2018, 8, 260. [CrossRef]

7. Zhang, J.W.; Tan, X.J.; Zheng, P.B. Non-destructive detection of wire rope discontinuities from residual magnetic field images using the hilbert-huang transform and compressed sensing. Sensors 2017, 17, 608. [CrossRef] [PubMed]

8. Tan, J.W. Principle and Technology of Steel Wire Rope Safety Detection; Science Press: Beijing, China, 2009.

9. Yang, S.Z.; Kang, Y.H.; Chen, H.G.; Yuan, J.M. Electromagnetic Nondestructive Testing of Wire Ropes; Machinery Industry Press: Beijing, China, 2017.

10. Gu, W.; Chu, J.X. A transducer made up of fluxgate sensors for testing wire rope defects. IEEE Trans. Instrum. Meas. 2002, 51, 120-124. [CrossRef]

11. Tan, X.J. Research on Non-Destructive Testing of Ferromagnetic Components with Weak Magnetic Imaging: In a Case of Wire Rope. Master's Thesis, Henan University of Science and Technology, Henan, China, 2018. 
12. Norouzi, E.; Ravanbod, H. Optimization of the flux distribution in magnetic flux leakage testing. Mater. Eval. 2010, 3, 360-364. [CrossRef]

13. Zhao, M.; Zhang, D.L. Magnetic flux leakage of typical defect of wire rope based on FE simulation. Nondestr. Test. 2009, 31, 177-180.

14. Lenard, S.; Atherton, D.L. Calculation of the effects of anisotropy on magnetic flux leakage detector signals. IEEE Trans. Magn. 1996, 32, 1905-1909. [CrossRef]

15. Krzywosz, K. Comparion of electromagnetic techniques for nondestructive inspetion of ferromagnetic tubing. Mater. Eval. 1990, 48, 42-45.

16. Kalwa, E.; Piekarski, K. Design of inductive sensors for magnetic testing of steel ropes. NDT E Int. 1991, 24, 328. [CrossRef]

17. Dutta, S.M.; Ghorbel, F.H.; Stanley, R.K. Simulation and analysis of 3-D magnetic flux leakage. IEEE Trans. Magn. 2009, 45, 1966-1972. [CrossRef]

18. Trevino, D.A.G.; Dutta, S.M.; Ghorbel, F.H.; Karkoub, M. An improved dipole model of 3-D magnetic flux leakage. IEEE Trans. Magn. 2016, 52, 1-7. [CrossRef]

19. Sun, Y.H.; Kang, Y.H. Magnetic mechanisms of magnetic flux leakage nondestructive testing. Appl. Phys. Lett. 2013, 103, 184104. [CrossRef]

20. Gao, G.H.; Lian, M.J.; Xu, Y.G.; Qin, Y.N.; Gao, L. The effect of variable tensile stress on the MFL signal response of defective wire ropes. INSIGHT 2016, 58, 135-141. [CrossRef]

21. Coramik, M.; Ege, Y. Discontinuity inspection in pipelines: A comparison review. Measurement 2017, 111, $359-373$. [CrossRef]

22. Kang, Y.H.; Li, Z.J.; Yang, Y.; Qiu, C. Mini-micro sensor and device for wire rope MFL testing. Nondestr. Test. 2014, 36, 11-15.

23. Wang, H.Y.; Hua, G.; Tian, J. Research on detection device for broken wires of coal mine-hoist cable. J. Chin. Univ. Mini. Technol. 2007, 17, 376-381. [CrossRef]

24. Jomdecha, C.; Prateepasen, A. Design of modified electromagnetic main-flux for steel wire rope inspection. NDT E Int. 2009, 42, 77-83. [CrossRef]

25. Wang, H.Y.; Xu, Z.; Hua, G.; Tian, J.; Zhou, B.B.; Lu, Y.H.; Chen, F.J. Key technique of a detection sensor for coal mine wire ropes. Min. Sci. Technol. 2009, 19, 170-175. [CrossRef]

26. Song, K.; Chen, C.; Kang, Y.H.; Li, J.J.; Ren, J.L. Mechanism study of AC-MFL method using U-shape inducer. Chin. J. Sci. Instrum. 2012, 33, 1980-1985. [CrossRef]

27. Yan, X.L.; Zhang, D.L.; Pan, S.M.; Zhao, E.C.; Gao, W. Online nondestructive testing for fine steel wire rope in electromagnetic interference environment. NDT E Int. 2017, 92. [CrossRef]

28. Kaur, A.; Gupta, A.; Aggarwal, H.; Arora, K.; Garg, N.; Sharma, M.; Sharma, S.; Aggarwal, N.; Sapra, G.; Goswamy, J.K. Non-destructive evaluation and development of a new wire rope tester using parallely magnetized NdFeB magnet segments. J. Nondestr. Eval. 2018, 37, 61. [CrossRef]

29. Sun, Y.H.; Wu, J.B.; Feng, B.; Kang, Y.H. An opening electric-mfl detector for the ndt of in-service mine hoist wire. IEEE Sens. J. 2014, 14, 2042-2047. [CrossRef]

30. Liu, X.C.; Xiao, J.W.; Wu, B.; He, C.F. A novel sensor to measure the biased pulse magnetic response in steel stay cable for the detection of surface and internal flaws. Sens. Actuators A Phys. 2018, 269, 218-226. [CrossRef]

31. Yan, X.L.; Zhang, D.L.; Zhao, F. Improve the signal to noise ratio and installation convenience of the inductive coil for wire rope nondestructive testing. NDT E Int. 2017, 92, 221-227. [CrossRef]

32. Fedorko, G.; Molnár, V.; Ferková, Ž.; Peterka, P.; Krešák, J.; Tomašková, M. Possibilities of failure analysis for steel cord conveyor belts using knowledge obtained from non-destructive testing of steel ropes. Eng. Fail. Anal. 2016, 67, 33-45. [CrossRef]

33. Sun, Y.H.; Liu, S.W.; Li, R.; Ye, Z.J.; Kang, Y.H.; Chen, S.B. A new magnetic flux leakage sensor based on open magnetizing method and its on-line automated structural health monitoring methodology. Struct. Health Monit. 2015, 14, 583-603. [CrossRef]

34. Xu, F.Y.; Wang, X.S.; Wu, H.T. Inspection method of cable-stayed bridge using magnetic flux leakage detection: Principle, sensor design, and signal processing. J. Mech. Sci. Technol. 2012, 26, 661-669. [CrossRef]

35. Wang, H.T.; Tian, J.; Meng, G.Y. A sensor model for defect detection in mine hoisting wire ropes based on magnetic focusing. INSIGHT 2017, 59, 143-148. [CrossRef] 
36. Kim, J.W.; Park, S. Magnetic flux leakage sensing and artificial neural network pattern recognition-based automated damage detection and quantification for wire rope non-destructive evaluation. Sensors 2018, 18, 109. [CrossRef]

37. Tian, J.; Wang, H.Y. Research on magnetic excitation model of magnetic flux leakage for coal mine hoisting wire rope. Adv. Mech. Eng. 2015, 7, 1-11. [CrossRef]

38. Zhou, J.Y.; Tian, J.; Wang, H.Y.; Li, Y.M.; Wu, M. Numerical simulation of magnetic excitation based on a permanent magnet co-directions array sensor. INSIGHT 2018, 60, 568-574. [CrossRef]

39. Singh, W.S.; Rao, B.P.C.; Mukhopadhyay, C.K.; Jayakumar, T. GMR-based magnetic flux leakage technique for condition monitoring of steel track rope. INSIGHT 2011, 53, 377-381. [CrossRef]

40. Liu, X.C.; Wang, Y.J.; Wu, B.; Gao, Z.; He, C.F. Design of tunnel magnetoresistive-based circular MFL sensor array for the detection of flaws in steel wire rope. J. Sens. 2016, 2016. [CrossRef]

41. Lu, T.; Zhang, L.H. Wire Rope Inspector; China University of Mining and Technology Press: Xuzhou, China, 2007; pp. 74-78.

42. Zhou, P.; Zhou, G.B.; He, Z.Z.; Tang, C.Q.; Zhu, Z.C.; Li, W. A novel texture-based damage detection method for wire ropes. Measurement 2019. under review.

43. Vallan, A.; Molinari, F. A vision-based technique for lay length measurement of metallic wire ropes. IEEE Trans. Instrum. Meas. 2009, 58, 1756-1762. [CrossRef]

44. Yaman, O.; Karakose, M. Auto-correlation based elevator rope monitoring and fault detection approach with image processing. In Proceedings of the 2017 International Artificial Intelligence and Data Processing Symposium (IDAP), Malatya, Turkey, 16-17 September 2017.

45. Sun, H.X.; Zhang, Y.H.; Luo, F.L. Texture segmentation and boundary recognition of wire rope images in complicated background. Acta Photonica Sinica 2010, 39, 1666-1671. [CrossRef]

46. Platzer, E.S.; Nägele, J.; Wehking, K.H.; Denzler, J. HMM-based defect localization in wire ropes-a new approach to unusual subsequence recognition. Pattern Recognition. In Proceedings of the Dagm Symposium, Jena, Germany, 9-11 September 2009; pp. 442-451.

47. Platzer, E.S.; Wehking, K.H.; Denzler, J. On the suitability of different features for anomaly detection in wire ropes. In Proceedings of the International Conference on Computer Vision, Imaging and Computer Graphics, Lisboa, Portugal, 5-8 February 2009; pp. 296-308.

48. Wacker, E.S.; Denzler, J. Enhanced anomaly detection in wire ropes by combining structure and appearance. Pattern Recognit. Lett. 2013, 34, 942-953. [CrossRef]

49. Wacker, E.S.; Denzler, J. An analysis-by-synthesis approach to rope condition monitoring. In Proceedings of the International Symposium on Visual Computing, Las Vegas, NV, USA, 29 November-1 December 2010; pp. 459-468.

50. Sanchez-Brea, L.M.; Siegmann, P.; Rebollo, M.A.; Bernabeu, E. Optical technique for the automatic detection and measurement of surface defects on thin metallic wires. Appl. Opt. 2000, 39, 539-545. [CrossRef] [PubMed]

51. Laguerre, L.; Treyssede, F. Non-destructive evaluation of seven-wire strands using ultrasonic guided waves. Eur. J. Environ. Civil Eng. 2011, 15, 487-500. [CrossRef]

52. Raisutis, R.; Kazys, R.; Mazeika, L.; Zukauskas, E.; Samaitis, V.; Jankauskas, A. Ultrasonic guided wave-based testing technique for inspection of multi-wire rope structures. NDT E Int. 2014, 62, 40-49. [CrossRef]

53. Liu, Z.H.; Zhao, J.C.; Wu, B.; Zhang, Y.N.; He, C.F. Configuration optimization of magnetostrictive transducers for longitudinal guided waves inspection in seven-wire steel strands. NDT E Int. 2010, 43, 484-492. [CrossRef]

54. Treyssède, F.; Laguerre, L. Investigation of elastic modes propagating in multi-wire helical waveguides. J. Sound Vib. 2010, 329, 1702-1716. [CrossRef]

55. Vanniamparambil, P.A.; Khan, F.; Hazeli, K.; Cuadra, J.; Schwartz, E.; Kontsos, A.; Bartoli, I. Novel optico-acoustic nondestructive testing for wire break detection in cables. Struct. Control Health Monit. 2013, 1319-1350. [CrossRef]

56. $\mathrm{Xu}, \mathrm{J} . ; \mathrm{Wu}, \mathrm{X} . \mathrm{J}$;; Sun, P.F. Detecting broken-wire flaws at multiple locations in the same wire of prestressing strands using guided waves. Ultrasonics 2013, 53, 150-156. [CrossRef]

57. Raisutis, R.; Kazys, R.; Mazeika, L.; Samaitis, V.; Zukauskas, E. Propagation of ultrasonic guided waves in composite multi-wire ropes. Materials 2016, 9, 451. [CrossRef]

58. Tse, P.W.; Rostami, J. Advanced signal processing methods applied to guided waves for wire rope defect detection. In AIP Conference Proceedings; AIP Publishing: College Park, MD, USA, 2016; Volume 1706, p. 030006.

59. Li, H.; Wang, W.T.; Zhou, W.S. Fatigue damage monitoring and evolution for basalt fiber reinforced polymer materials. Smart Struct. Syst. 2014, 14, 307-325. [CrossRef] 
60. Hoon, S.; Gyuhae, P.; Jeannette, R.W.; Nathan, P.L.; Charles, R.F. Wavelet-based active sensing for delamination detection in composite structures. Smart Mater. Struct. 2003, 13, 153. [CrossRef]

61. Drummond, G.; Watson, J.F.; Acarnley, P.P. Acoustic emission from wire ropes during proof load and fatigue testing. NDT E Int. 2007, 40, 94-101. [CrossRef]

62. Bai, W.; Chai, M.; Li, L.; Li, Y.; Duan, Q. Acoustic emission from elevator wire ropes during tensile testing. In Advances in Acoustic Emission Technology: Proceedings of the World Conference on Acoustic Emission-2013; Springer: New York, NY, USA, 2013; pp. 217-224.

63. Casey, N.F.; Taylor, J.L. Acoustic emission of steel wire ropes. Wire Ind. 1984, 51, 79-82.

64. Casey, N.F.; Taylor, J.L. Evaluation of wire ropes by AE techniques. Brit. J. Non. Destruct. Test. 1985, 27, 351-356.

65. Casey, N.F.; Wedlake, D.; Taylor, J.L.; Holford, K.M. Acoustic detection of wire rope failure. Wire Ind. 1985, 52, $307-309$.

66. Casey, N.F.; Taylor, J.L.; Holford, K.M. Wire break detection during tensile fatigue testing of $40 \mathrm{~mm}$ wire rope. Brit. J. Non. Destruct. Test. 1985, 30, 338-341.

67. Casey, N.F.; White, H.; Taylor, J.L. Frequency analysis of the signals generated by the failure of constituent wires of a wire rope. NDT E Int. 1989, 56, 583-586. [CrossRef]

68. Casey, N.F.; Laura, P.A.A. A review of the acoustic-emission monitoring of wire rope. Ocean Eng. 1997, $24,935-947$. [CrossRef]

69. Shao, Y.B.; Yu, D.; Wang, S.; Zhu, Z.M.; Yin, W.Q. Quantitative method to detect wire break in wire rope by acoustic emission techniques. J. Northeast. Univ. Nat. Sci. 1999, 20, 130-132.

70. Ding, Y.; Reuben, R.L.; Steel, J.A. A new method for waveform analysis for estimating AE wave arrival times using wavelet decomposition. NDT E Int. 2004, 37, 279-290. [CrossRef]

71. Li, S.; Wu, Y.; Shi, H. A novel acoustic emission monitoring method of cross-section precise localization of defects and wire breaking of parallel wire bundle. Struct. Control Health Monit. 2019, 26, e2334. [CrossRef]

72. Li, H.; Huang, Y.; Chen, W.L.; Ma, M.L.; Tao, D.W.; Ou, J.P. Estimation and warning of fatigue damage of FRP stay cables based on acoustic emission techniques and fractal theory. Comput. Aided Civil Infrastruct. Eng. 2011, 26, 500-512. [CrossRef]

73. Cao, Q.S.; Liu, D.; Zhou, J.H.; Zhou, J.M. Non-destructive and quantitative detection method for broken wire rope. Chin. J. Sci. Instrum. 2011, 32, 787-794. [CrossRef]

74. Cao, Q.S.; Liu, D.; He, Y.H.; Zhou, J.H.; Codrington, J. Nondestructive and quantitative evaluation of wire rope based on radial basis function neural network using eddy current inspection. NDT E Int. 2012, 46, 7-13. [CrossRef]

75. Zhang, Y.C.; Xu, G.Y.; Cui, J. System used to detect steel wire rope based on X-ray digital imaging technology. Image Technol. 2008, 2, 33-39.

76. Peng, P.C.; Wang, C.Y. Use of gamma rays in the inspection of steel wire ropes in suspension bridges. NDT E Int. 2015, 75, 80-86. [CrossRef]

77. Cao, Y.N.; Zhang, D.L.; Xu, D.G. The state-of-art of quantitative nondestructive testing of wire ropes. Nondestruct. Test. 2005, 27, 91-95.

78. Wu, P.; Hua, H.Y. Discussion on the existing problems of steel wire rope nondestructive testing. Nondestruct. Test. 2017, 39, 65-68. [CrossRef]

79. Zhou, P.; Zhou, G.B.; Zhu, Z.C.; Tang, C.Q.; He, Z.Z.; Li, W.; Jiang, F. Health monitoring for balancing tail ropes of a hoisting system using a convolutional neural network. Appl. Sci. 2018, 8, 1346. [CrossRef]

80. Guo, X.J.; Chen, L.; Shen, C.Q. Hierarchical adaptive deep convolution neural network and its application to bearing fault diagnosis. Measurement 2016, 93, 490-502. [CrossRef]

81. Zhao, R.; Yan, R.Q.; Chen, Z.H.; Mao, K.Z.; Wang, P.; Gao, X. Deep learning and its applications to machine health monitoring. Mech. Syst. Signal Process. 2019, 115, 213-237. [CrossRef]

82. Guo, X.J.; Shen, C.Q.; Chen, L. Deep fault recognizer: An integrated model to denoise and extract features for fault diagnosis in rotating machinery. Appl. Sci. 2017, 7, 41. [CrossRef]

83. Zhou, P.; Zhou, G.B.; He, Z.Z.; Zhu, Z.C.; Tang, C.Q.; Li, W. A novel deep learning-based damage monitoring and diagnosis method for wire ropes. Mech. Syst. Signal Process. 2019. under review.

(C) 2019 by the authors. Licensee MDPI, Basel, Switzerland. This article is an open access article distributed under the terms and conditions of the Creative Commons Attribution (CC BY) license (http://creativecommons.org/licenses/by/4.0/). 